\title{
REVIEW
}

\section{More targets, more pathways and more clues for mutant p53}

\author{
S Garritano ${ }^{1}$, A Inga ${ }^{2}$, F Gemignani ${ }^{1}$ and S Landi ${ }^{1}$
}

Mutations in the transcription factor p53 are among the most common genetic alterations in human cancer, and missense p53 mutations in cancer cells can lead to aggressive phenotypes. So far, only few studies investigated transcriptional reprogramming under mutant p53 expression as a means to identify deregulated targets and pathways. A review of the literature was carried out focusing on mutant p53-dependent transcriptome changes with the aims of (i) verifying whether different p53 mutations can be equivalent for their effects, or whether there is a mutation-specific transcriptional reprogramming of target genes,

(ii) understanding what is the main mechanism at the basis of upregulation or downregulation of gene expression under the p53 mutant background, (iii) identifying novel candidate target genes of WT and/or mutant p53 and (iv) defining cellular pathways affected by the mutant p53-dependent gene expression reprogramming. Nearly 600 genes were consistently found upregulated or downregulated upon ectopic expression of mutant p53, regardless of the specific p53 mutation studied. Promoter analysis and the use of ChIP-seq data indicate that, for most genes, the expression changes could be ascribed to a loss both of WT p53 transcriptional activation and repressor functions. Pathway analysis indicated changes in the metabolism/catabolism of amino acids such as aspartate, glutamate, arginine and proline. Novel p53 candidate target genes were also identified, including ARID3B, ARNT2, CLMN, FADS1, FTH1, KPNA2, LPHN2, PARD6B, PDE4C, PIAS2, PRPF4OA, PYGL and RHOBTB2, involved in the metabolism, xenobiotic responses and cell differentiation.

Oncogenesis (2013) 2, e54; doi:10.1038/oncsis.2013.15; published online 1 July 2013

Subject Categories: Cancer metabolism

Keywords: p53; microarray; target genes

\section{INTRODUCTION}

The tumor suppressor p53 is a 393-amino-acid nuclear phosphoprotein that responds to numerous stress stimuli, including DNA damage ${ }^{1}$ and hypoxia. ${ }^{2}$ Following homotetramerization, it acts as a transcription factor $^{3}$ and modulates the expression of a variety of genes, leading to enhanced DNA repair, control of cell cycle and apoptosis, and maintaining cellular homeostasis. ${ }^{4-6}$ The p53 targets are only partially known, with assessments suggesting their number to be nearly 2000 genes. $^{7}$

CDKN1A, MDM2, BAX, GADD45 and $B B C 3$ are paradigmatic examples of upregulated target genes where $\mathrm{p} 53$ exerts its activity via evolutionarily conserved cis-response elements (p53RE). ${ }^{8}$ The importance of $\mathrm{p} 53$ for the biology of cancer is evident by the fact that colorectal, breast and most other human solid tumors show a high frequency of somatic mutations within the TP53 gene (www.iarc.fr/p53). Moreover, germline mutations within TP53 cause the Li-Fraumeni syndrome, a dominantly inherited cancer proneness syndrome with an elevated risk of developing adrenocortical carcinoma, choroid plexus carcinomas, sarcomas and other types of cancer in multiple sites at a young age. ${ }^{9}$ So far, nearly 2000 different single amino-acid changes in p53 have been reported in tumors, ${ }^{10}$ and their frequencies vary markedly: next to exceedingly rare mutations, strong hotspots are evident. ${ }^{11,12}$ This latter group of mutations affects, in particular, codons 175, 248, 249,273 or 282 . The impact of mutations on p53 functions can vary from a wild-type-like activity, for example, the $\mathrm{R} 337 \mathrm{H}$ mutations associated with predisposition to adrenocortical carcinoma, ${ }^{13}$ to a partial function or to a suspected complete loss of function (LOF). ${ }^{12,14}$
According to Resnick et al., different mutant p53s retaining a partial activity (for example, T123A or S215C) show specific effects on the transactivation of target promoters, leading to mutationspecific altered regulation of hundreds of genes (the 'piano model'), resulting in a variety of biological consequences. ${ }^{15,16}$ Cells can show lack of control of their cell cycle and weakened apoptosis and DNA repair. However, in selected examples, separation of p53 functions was observed, with defective apoptotic control, but wild-type function in cell cycle arrest. ${ }^{17}$ Moreover, knockin mouse models showed varied phenotypes, suggesting the occurrence of mutationspecific gene expression reprogramming also in vivo. ${ }^{18,19}$ However, most studies related to mutant p53 activity were performed on hotspot mutations, using reconstituted assays ${ }^{12,14,20}$ or other in vitro models. Following these experimentations, it was observed that hotspot mutations have the least transactivating activity of common targets and therefore they were suggested to cause a p53 LOF. Hotspot p53 mutations were reported to be associated with more aggressive malignancies and could confer novel phenotypes in vivo, including an increased metastatic capacity and resistance to chemotherapies. $^{21-27}$ The acquired phenotypes of specific mutant p53s are generally referred to as gain-of-function properties, ${ }^{28}$ but it is unclear if these features are restricted to or distinct among specific p53 hotspot mutations. Examining the impact of hotspot p53 mutations at a transcriptome level, a large number of genes are downregulated. However, there are also a restricted number of WTp53 targets whose transactivation seems not to be hampered by p53 mutations. ${ }^{7}$ Moreover, there are also genes that are upregulated under mutant but not WT p53 expression. It is not clear whether different mutants can lead to similar transcriptional changes or have

'Department of Biology, University of Pisa, Pisa, Italy and ${ }^{2}$ Laboratory of Transcriptional Networks, Centre for Integrative Biology, CIBIO, University of Trento, Trento, Italy. Correspondence: Professor S Landi, Department of Biology, University of Pisa, via Derna, 1, Pisa 56100, Italy.

E-mail: slandi@biologia.unipi.it

Received 28 February 2013; accepted 20 April 2013 
different impact on it (like an extension of the 'piano model') and whether the gained phenotypes can be related to specific genes upregulated in the p53 mutant background. Thus, in this work, the focus was placed on cancer-associated p53 hotspot mutations that exhibit a loss of transactivation function in reconstituted assays, ${ }^{12,14}$ and a review of the literature was performed, with the following aims: (1) to verify whether different p53 mutations can be equivalent for their effects, or whether there is a mutation-specific transcriptional reprogramming of target genes, (2) to understand what is the main mechanism at the basis of upregulation or downregulation of gene expression under the p53 mutant background, (3) to identify the novel candidate target genes of WT and/or mutant p53 and (4) to define cellular pathways affected by the mutant $\mathrm{p} 53$-dependent gene expression reprogramming.

\section{SELECTION OF THE PUBLISHED LITERATURE}

In order to identify genes differentially modulated upon the expression of mutant p53, only potentially unbiased transcriptome studies published in the literature were collected. In fact, in transcriptome studies, target genes are analyzed without formulating any a priori hypothesis and, virtually, all the genes are evaluated with the same relevance. An extensive literature search was carried out using PubMed (http://www.ncbi.nlm.nih.gov/ pubmed) to collect original papers. Articles were selected by screening title, abstract and full text, and only those reporting the effects of ectopic expression of p53 mutants on the transcriptome were considered further.

Out of over 2000 known p53 mutations reported by the IARC (www.iarc.fr/p53) or UMD TP53 databases, ${ }^{10,29}$ only 12, falling in 11 different codon sites (Figure 1), were studied through global gene expression changes. Those 11 mutated codons lie within the sequence-specific DNA-binding domain, correspond to hotspot mutations in tumors and result in LOF in functional assays. Studies on p53-dependent transcriptomes were few and heterogeneous in their experimental design, with a variable p53 status of the cell lines used, thus limiting the strength of the comparisons. Therefore, conservatively, conclusions on mutant p53-dependent gene deregulation were drawn only when at least three independent p53 mutations showed a coherent effect on the same target. Now on, genes upregulated under the ectopic expression of at least three different mutant p53 genetic backgrounds are defined as UMB, whereas DMB are the genes downregulated under at least three different mutant p53s. The results were obtained relying on the statistical analyses imbedded within each study, and a list of differentially expressed genes was compiled for further analysis (Supplementary Data S1). For each chosen article, in Table 1, the p53 missense mutation studied, and the cell lines used to perform the experiments were reported.

\section{IN SILICO ANALYSES OF PROMOTERS AND PATHWAYS}

COMPASSS (COMplex PAttern of Sequence Search Software), ${ }^{30}$ a software that allows to perform custom pattern searches in entire genomes, was used to analyze the promoters. Given that most of the deregulated genes are not well-established p53 target genes, the focus was placed on the identification of non-canonical p53REs, ${ }^{31,32}$ particularly a half-site RE motif. In the exploratory search, a conservative approach was used by limiting inspection to 2-kb upstream of annotated transcriptional start sites, not allowing mismatches in the half-site decameric motif, and requiring the presence of a cluster of at least two half-sites within one nucleosome. ${ }^{32}$ Hence, the following input were used: RRRCWWGYYY( $\left.\mathrm{N}_{0-50}\right)$ RRRCWWGYYY and NRRCWWGYYN $\left(\mathrm{N}_{0-}\right.$ $\left.{ }_{50}\right)$ NRRCWWGYYN. Two closely spaced p53 half-sites either in a direct orientation (RRRCWRRRCW) or lacking the CWWG core (WGYYYRRRCW), or having a relaxed motif definition (RRNCNNGNYN) (all sequence features that have been associated with genes repressed by WTp53), ${ }^{8}$ were also queried. Thus, COMPASSS was used to analyze the promoters of $U M B$ and $D M B$ genes and to measure the 'baseline' number of p53REs found in the whole human genome. Then, a binomial distribution-based statistics (approximated as normal distribution) was used, in order to verify whether the promoters were enriched for the input motifs, as compared with the baseline level.

The complex pattern of gene transcription changes was further analyzed with the tool Database for Annotation, Visualization and Integrated Discovery, in order to detect whether mutations within p53 could affect specific biological pathways. ${ }^{33}$ Database for Annotation, Visualization and Integrated Discovery uses all the human genes as background to perform the comparisons, and if a group of genes is enriched within a specific biological process or pathway, the $P$-value of the modified Fisher's exact test will be lower than the cutoff (0.05). First, the short lists of $U M B$ and $D M B$ genes, either separated or combined, were used as input, but the total number of genes was not large enough to obtain statistically significant results. Thereafter, the analyses were repeated with a broadened input list, that is, the list of genes changing their expression under at least one p53 mutant background (that is, those reported in Supplementary Table S1).

\section{RESULTS AND DISCUSSION}

Similar deregulation profile of genes by distinct p53 mutation hotspots

By observing $U M B$ and $D M B$ genes, consistent trends emerged (Table 2, see also Supplementary Table S1). A total of 401 genes were found downregulated under the ectopic expression of at least three different mutant p53s, whereas 260 genes were found upregulated (reported in Tables 3 and 4, respectively). Given the

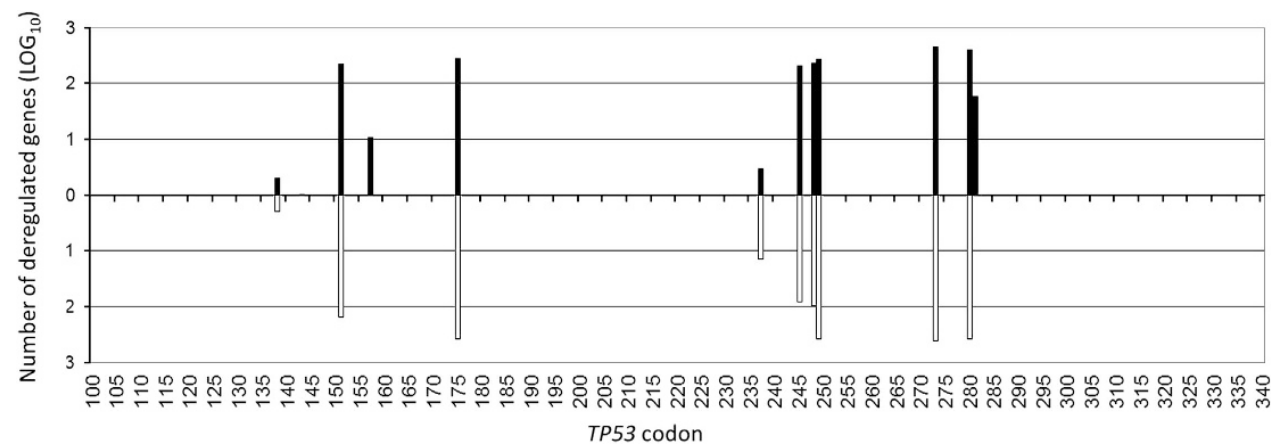

Figure 1. Number of genes (Log10) upregulated (black bars) and downregulated (white bars) following in vitro studies where a mutant form of p53 is overexpressed. Only few codons were assayed and for each mutation it is shown that the number of genes going overexpressed is approximately similar to those downregulated. The missense mutations falling within the codon 248 (R248Q and R248W) were considered as a unique one, in order to empower the study. 
Table 1. List of selected article

\begin{tabular}{lcc}
\hline Article & Cells used & Mutation tested \\
\hline 88 & TKS, WTK1 & M237I \\
89 & HCT 116 & A138P; R175H \\
90 & H1299 & R175H \\
91 & HME1 & R175H; R273H; R280K; R249S \\
65 & H1299 & R175H; R273H; D281G \\
92 & U2OS & R175H; V157F; R248Q \\
93 & LNCaP & G245S; R248W; R273H; R273C \\
94 & H1299 & R175H; R248W; R273H; D281G \\
95 & H1299 & D281G \\
96 & H1299 & R175H; R273H; D281G \\
\hline
\end{tabular}

For each article, the investigated p53 missense mutations and the cell lines used to perform the experiments are reported.

heterogeneity among studies, it is likely that consistent findings reveal true p53 target genes. Similar, although less confident, results could be obtained when the expression of genes was evaluated comparing at least two p53 mutants: 446 genes were found to be downregulated, whereas 503 genes were found to be upregulated. Thus, overall, the gene expression reprogramming did not seem to differ in relation to the mutant p53 hotspot analyzed. However, it should be also noticed that a small share of genes (48 out of 1846, 2.6\%) was described as behaving discordantly, in relation to the p53 mutant assayed. It should be acknowledged that a systematic comparison of all mutant p53s under the same experimental conditions was not found in the literature, and thus subtle mutation-specific differences cannot be ruled out.

Hypothesized mechanisms at the basis of DMB and UMB phenotypes

In order to better understand the possible mechanisms related to the changes of expression caused by mutations within $\mathrm{p} 53, D M B$ genes were first compared with the information from Riley's list, ${ }^{3}$ who reported 126 experimentally validated p53 target genes. These genes were crossed with those reported in Table 3 and 26 in common were found (bolded in Table 3). Almost all of them, 25, were genes normally activated by the WTp53. Then, COMPASSS was used (the detailed statistics are reported for each chromosome and for each p53RE motif in Supplementary Table 2) and it was observed that $D M B$ genes were enriched for p53RE motifs typically found in genes transactivated by WTp53. This was expected and was consistent with the comparison made with Riley's data. Thus, it is conceivable that, for most of the $D M B$ genes, the lack of expression is related to the LOF of p53.

When Riley's list was compared with the UMB genes (Table 4), only three were in common (bolded), preventing to draw any conclusion. According to COMPASSS, UMB genes were specifically enriched for a pair of the p53RE variant motif (RRNCNNGNYN) that was previously related to WTp53-dependent gene repression. ${ }^{8}$ Out of $260 U M B$ genes, 242 contained a putative repressor element. It is, however, important to note that the p53RE variant pattern search may retrieve false-positive results. This motif was also enriched over the baseline for the $D M B$ genes (this because it represents a more degenerated version of the canonical p53RE) confirming the difficulty in separating p53-upregulated and p53-downregulated genes purely on the basis of the cis-regulatory elements. ${ }^{3,8,32}$ The fact that WTp53 could bind p53RE within specific UMB genes is reinforced by studies of chromatin immunoprecipitation followed by DNA sequencing (ChiP-seq) coupled to transcriptome analysis. ${ }^{34}$ In fact, high-confidence p53 occupancy sites have been mapped not only for $57 D M B$ genes but also for $23 U M B$ genes (underlined in Tables 3 and 4). In summary, also UMB genes could be explained with the loss of activity, that is, a loss of transcriptional repression,
Table 2. Number of genes upregulated and downregulated following in vitro studies where a mutant form of p53 is overexpressed

\begin{tabular}{|cccc}
\hline $\begin{array}{c}\text { Number } \\
\text { of genes }\end{array}$ & $\begin{array}{c}\text { \% Of the } \\
\text { total } \\
\text { number } \\
\text { of genes }\end{array}$ & $\begin{array}{c}\text { Number of mutations } \\
\text { on different } p 53 \\
\text { codons, leading to } \\
\text { upregulation }\end{array}$ & $\begin{array}{c}\text { Number of mutations } \\
\text { on different } p 53 \\
\text { codons, leading to } \\
\text { downregulation }\end{array}$ \\
\hline 151 & $8.2 \%$ & 0 & 1 \\
698 & $37.8 \%$ & 1 & 0 \\
446 & $24.2 \%$ & 0 & $>1$ \\
503 & $27.2 \%$ & $>1$ & 0 \\
401 & $22 \%$ & 0 & 03 \\
260 & $14 \%$ & $\geqslant 3$ & 0 \\
Inconsistent & & & $\geqslant 1$ \\
48 & $2.6 \%$ & $\geqslant 1$ & \\
\hline
\end{tabular}

Only 48 out of 1846 were described as behaving differently according to the p53 mutated codon assayed. The greatest majority of genes (949) showed a reproducible upregulation or downregulation when various p53 mutations were assayed.

toward specific targets. Actually, it was shown recently that $\mathrm{p} 53$ bears a repressor activity for genes such as CHEK1, BCL2, ARF and FOS, ${ }^{8}$ MDR1 and Lasp1. ${ }^{8,35}$ Moreover, experiments in the yeast assays showed that mutant p53s lose the transactivating capability towards several target REs from target genes. ${ }^{12,14,20}$

Three generally accepted mechanisms of direct p53-mediated transcriptional repression are known: (1) steric interference by masking overlapping transcription factor binding sites, ${ }^{36,37}$ (2) sequestration of transcription activators ${ }^{38}$ and (3) recruitment of histone deacetylases. ${ }^{3,39}$ Moreover, other indirect mechanisms were suggested, such as the transcriptional activation of microRNAs, known inhibitors of mRNA translation and stability. ${ }^{39,40}$ Thus, a 'full-loss-of-function hypothesis' could explain both the $U M B$ and the $D M B$ genes. However, alternatives are discussed further in final remarks section.

\section{Novel targets for WTp53}

Previous analyses were also useful to detect novel putative direct p53 targets. In fact, a short list of highly likely candidate p53 targets was obtained applying the in silico analysis of p53REs within the $D M B$ and $U M B$ genes, crossed with the results from a ChIP-seq study. ${ }^{34}$ In Supplementary Table S3, all the UMB and DMB genes positive for a p53REs within the promoter (through COMPASSS) were listed. Following the cross with the ChIP-seq study, known p53 targets were found (including ATF3, BTG2, BTG3, MYC, CDKN1A, ENC1, TP53/3 and TP53INP1). However, interestingly, a restricted number of novel potential p53 targets were also suggested. These are: $A R I D 3 B$, ARNT2, CLMN, FADS1, FTH1, KPNA2, LPHN2, PARD6B, PDE4C, PIAS2, PRPF4OA, PYGL and RHOBTB2. Intriguingly, some of them, belonging to the UMB category, were shown to be in causal relationship with features of the malignant phenotype and their upregulation in tumor correlates with a worsening of the prognosis. For example, an overexpression of $A R I D 3 B$ in human neuroblastoma cell lines is more common in stage IV neuroblastoma than in stages I-III, indicating its role in the progression of malignant neuroblastoma. ${ }^{41}$ The upregulation of ARNT2 is also common in neuronal-derived tumors. ARNT2 forms complexes with HIF-1a (Hypoxia-inducible factor 1-alpha), and it allows for initiating hypoxia/nutrient deprivation-induced vascular endothelial growth factor expression, therefore permitting tumor angiogenesis. ${ }^{42}$ FTH1 was found to be overexpressed in tumorspheres, and its upregulation has an important anti-apoptotic role. ${ }^{43,44}$ Moreover, FHIT overexpression was shown to have a role in increasing the multidrug resistance of cancer cells, ${ }^{45}$ whereas its silencing caused an increased sensitivity. ${ }^{46}$ KPNA2 was found to be highly expressed in different types of cancer, 
Table 3. List of genes consistently downregulated where at least three independent mutations were assayed

\begin{tabular}{|c|c|c|c|c|c|c|c|c|c|c|c|c|}
\hline ABAT & C15orf41 & $C O B L$ & EDNRB & GABARAPL 1 & GTPBP2 & KIAA1211 & MARVELD2 & $P 9$ & $P S A$ & SHC4 & TBXA2R & VCAN \\
\hline$A B C A 12$ & C16orf5 & COL18A1 & EFS & GAD1 & $\mathrm{H} 19$ & KIAA1324 & MCC & PADI3 & PSEN2 & SHROOM2 & TEAD3 & VSNL1 \\
\hline$\overline{A B C G 1}$ & C17orf103 & COL2A1 & EGR2 & GADD45A & $H A G H$ & KIAA1751 & $\overline{M D F} I C$ & РАК6 & PSTPIP2 & SI & TFAP2E & WDR8 \\
\hline ABHD15 & C19orf59 & $C P E$ & ENC1 & $\overline{G A G E B 1}$ & HES2 & KITLG & MDM2 & PALMD & $\overline{\text { PTGES }}$ & $S L C 2 A 13$ & TGFBR1 & WFDC5 \\
\hline ABHD4 & C1orf187 & CPN2 & $\overline{E P B 49}$ & GALE & $\overline{H H A T L}$ & $\overline{K L R K 1}$ & $\overline{M E F 2 A}$ & PARD6G & PTPN22 & $S L C 2 A 8$ & TINAGL1 & WNT5A \\
\hline ABHD6 & C2orf3 & CRISPLD2 & EPHB4 & GAMT & HIC2 & KREMEN2 & MGC16703 & PARP10 & PVRL4 & $S L C 2 A 9$ & TINP1 & WWP1 \\
\hline ABTB2 & C4A & CRYAB & $\overline{E P P K 1}$ & GATA3 & HIST1H2AE & KRT78 & MGC4248 & PARP14 & PYHIN1 & SLC35D1 & TLR3 & XLalphas \\
\hline$A C P P$ & C4orf18 & CSNK1G1 & ETNK1 & GCH1 & $H L A-D M B$ & KRTAP2-1 & MIB2 & PCBP4 & RALGDS & SLC35E4 & TM7SF3 & YPEL3 \\
\hline$\overline{A H S A} 2$ & $\overline{c 50 r f 4}$ & $\overline{\text { CST1 }}$ & $\overline{E T V 7}$ & GCLC & HOXC13 & $K S R$ & MICALL2 & PCLO & $\overline{R A S A L T}$ & SLC39A8 & $\overline{T M E M 144}$ & ZBTB1 \\
\hline$\overline{A I F M 2}$ & C6orf204 & CST11 & $F 2 R L 2$ & GDAP1L1 & HOXD10 & LAMP3 & MIR & PDE3A & $\overline{R A S G E F} 1 B$ & SLC4A11 & TMEM27 & ZMAT3 \\
\hline AK1 & C7orf10 & CTSH & FAM105A & GDF15 & HSDL2 & LCE1B & MLF2 & PDE4C & RASSF6 & SLC4A4 & TMEM63B & $\overline{Z \text { ZNF197 }}$ \\
\hline AKR1B10 & C7orf57 & CYFIP2 & FAM134B & $\overline{\text { GDF9 }}$ & HSPA5BP1 & LDLRAP1 & $M M E$ & $\overline{P D E 4 D}$ & $\mathrm{RDH} 10$ & SLC9A1 & TMG4 & ZNF236 \\
\hline$\overline{A L D H 7 A 1}$ & C9orf100 & CYLC1 & FAM13C & GGT1 & HTR2A & LHX3 & MPZL2 & PENK & RET & SLC9A3R1 & TMTC3 & ZNF385A \\
\hline$A M A C R$ & C9orf98 & CYP2C9 & FAM43A & GGT6 & IDUA & LIN54 & MRAS & PERP & retll & SLCO2B1 & TNFRSF10B & ZNF441 \\
\hline ANK1 & $C A B C 1$ & CYP2S1 & FAM46C & GH2 & IFIT3 & LOC132671 & MRPL44 & PF4V1 & RGS12 & $\overline{S M A R C D} 3$ & $\overline{T N F R S F 14}$ & ZNF492 \\
\hline ANO4 & CABYR & СYРЗА7 & FAM69B & GHR & IFITM1 & LOC203274 & MSX1 & $P G F$ & $R H O D$ & SOM & TNP2 & ZNF746 \\
\hline APAF1 & CACNA2D2 & $\overline{D 4 S 234} E$ & FAM $82 A 1$ & GJC1 & IL24 & LOC283585 & MT1G & $\overline{P L A} 2 \mathrm{G} 2 \mathrm{~A}$ & RNASE7 & SORBS1 & TNRC6C & ZNF786 \\
\hline AРОВЕСЗС & CALML3 & $D D B 2$ & FAM87A & GLS & IL2RB & LOC284837 & MTMR6 & PLAC8 & RNF128 & SORL1 & TP53/11 & \\
\hline ARAP1 & CAPN1 & $\overline{D D C}$ & FBXO2 & GLS2 & INPP1 & LOC286434 & MYO6 & $\overline{P L A T}$ & RNF144B & SPAG1 & TP5313 & \\
\hline ARG1 & CARD18 & DFNB31 & FBXO22 & GM2A & INPP5D & LOC348938 & MYO7A & PLEK & RNF4 & SPN & $\overline{T P 53 I N P 1}$ & \\
\hline ARHGAP6 & CASP6 & DGKZ & $\overline{\text { FGF1 }}$ & GNA11 & ISG15 & LOC80154 & NADSYN1 & PLXNB3 & RPL36 & $S S B 2$ & TP53TG1 & \\
\hline ARHGEF3 & CBFA2T3 & DHRS2 & FITM2 & GNA14 & ISYNA1 & LRDD & NCRNA00085 & PMAIP1 & RPS27L & STAR & TP73 & \\
\hline$\overline{A S P A}$ & CCNA1 & $\overline{D I S P 1}$ & FLJ14312 & GNG2 & ITFG1 & LRP10 & $N E F L$ & $\overline{P O D X L}$ & $\overline{R P S 6 K A 1}$ & STARD5 & TRAF4 & \\
\hline ATP11B & ССТ6B & $\overline{D K K 2}$ & FLJ32065 & GPC1 & KAT2B & LTB4R & NHLH2 & POLH & RRM2B & STAT4 & TREM2 & \\
\hline$B C H E$ & $\mathrm{CDH} 10$ & DMRTC1 & FLJ36336 & GPR126 & $\overline{K C N B 1}$ & LUM & $\overline{N I F U}$ & $\overline{P O M Z P 3}$ & RTN1 & STOX2 & TRIM11 & \\
\hline$B C L 11 B$ & CDKN1A & DNAJC18 & FLJ40773 & GPR137B & KCNC4 & $M A B 21 L 1$ & NLRP1 & POU3F1 & $\overline{S A A 2}$ & SULF2 & TRIM2 & \\
\hline BDKRB1 & $\overline{C D K N 1 C}$ & DNAJC21 & FLNC & GPR155 & KCNJ12 & MAD1L1 & NOTCH1 & PPM1F & SAC3D1 & $\overline{S Y K}$ & TRIM22 & \\
\hline BDNF & CEACAM1 & DPYSL4 & FMN1 & GPR56 & KIAA0247 & $\overline{M A E L}$ & $\overline{N O T C H 3}$ & $P P P 2 R 2 B$ & SCN3B & SYNC & TRIM3 & \\
\hline BLNK & CES2 & DSG3 & FREQ & GPR87 & KIAA0284 & MAFB & NRCAM & $P P P 2 R 2 C$ & SCNN1G & SYTL2 & TSGA10 & \\
\hline BTF3L3 & CLCA2 & DUOX1 & FRMD8 & GRAMD2 & KIAA1026 & MAGEA4 & NRP2 & PRKX & SEC14L5 & TAF3 & TSPAN14 & \\
\hline BTG2 & CLDN19 & DUSP13 & FXYD2 & GREB1 & KIAA1052 & $M A P 2 K 3$ & OIP106 & PRKY & SERPINB5 & TAGLN & TSPY1 & \\
\hline C13orf31 & CNNM4 & $E D A R$ & $G 6 P C$ & GRN & KIAA1199 & MAPK13 & OTP & PRRX1 & SESN1 & TAP1 & ULBP2 & \\
\hline
\end{tabular}

The listed genes are reproducibly deregulated irrespectively on the mutated codon. Note that all the assayed mutations fall within the p53 DNA-binding domain. Bolded genes are in common with the functional assay proposed by Riley et al. ${ }^{3}$ Underlined are genes for which a high-confidence p53 occupancy sites had been mapped.

Table 4. List of genes consistently upregulated where at least three independent mutations were assayed

\begin{tabular}{|c|c|c|c|c|c|c|c|c|}
\hline ABLIM1 & CA9 & DRG1 & HIST1H4C & $L P P$ & MYO5B & ProSAPIP1 & SLCO4A1 & $X R C C 5$ \\
\hline ACTA2 & CARS & E2F3 & HMGB2 & LRRFIP1 & NAP1L1 & PRPF40A & SLPI & YARS \\
\hline ADAMTSL4 & CBR4 & E2F5 & HOMER 1 & LRRK1 & NARS & PRSS7 & SMA4 & ZBTB45 \\
\hline ADPRTL2 & CCNB1IP1 & EBAG9 & HOMER2 & $L Y N$ & NBEA & PRSS8 & SMTN & ZNF217 \\
\hline AGGF1 & CCNB2 & EFHA1 & HSP90AB1 & MAD2L1 1 & NDC80 & PVRL3 & SNTB2 & $\overline{Z N F 238}$ \\
\hline AKT2 & $\mathrm{CCNH}$ & EXPH5 & HYAL3 & MAL2 & NDUFA4L2 & PYGL & SPAG5 & ZNF24 \\
\hline ALDH1A3 & CCNL1 & $F 2 R$ & ID1 & MAP2K5 & NFATC2IP & $\overline{Q A R S}$ & SQSTM1 & ZNF273 \\
\hline$\overline{A L D H 2}$ & CD14 & FADS1 & $\overline{I D 3}$ & MAP4 & NFKBIA & RAD51C & SRM & ZNF415 \\
\hline$A L D H 3 A 1$ & CD44 & $\overline{\text { FAM169A }}$ & $\overline{I L 1} R L 1$ & MAPKAPK2 & NKTR & $\overline{R B B P 6}$ & SS18 & ZNF44 \\
\hline ANGPT1 & $C D C 2$ & FBXO31 & IMPDH2 & MAРКАРКЗ & $N L R X 1$ & RBBP8 & STAMBP & ZNF579 \\
\hline APS & $\overline{C D C 6}$ & FDFT1 & INADL & MAPKAPK4 & $N M I$ & RHOBTB2 & STAT3 & ZNF580 \\
\hline$A R$ & $\mathrm{CDH} 1$ & FECH & INF2 & MARCH_6 & NPC1 & $\overline{R H O G}$ & STATH & ZNF652 \\
\hline ARHGEF2 & $C D K L 3$ & FTH1 & INPPL1 & $M A R C K \bar{S}$ & NUP153 & RLN1 & TAF1A & \\
\hline$A R I D 3 B$ & CDS1 & $\overline{F Z D 3}$ & ITGA6 & $M A Z$ & $P A Q R 3$ & RNF44 & TARS & \\
\hline$\overline{A R N T 2}$ & CEBPB & GAGE3 & ITPR3 & MCL1 & PARD6B & RNF6 & TFAP2A & \\
\hline$\overline{A S B 13}$ & CKS1B & GAPVD1 & JUNB & МСM3 & PARP1 & RP3-402G11.5 & TMC6 & \\
\hline ATF3 & CLEC18C & GARS & JUP & MCM6 & PCGF2 & RPGRIP1 & TNFRSF9 & \\
\hline$\overline{A T I C}$ & CLMN & GHDC & KIAA0516 & ME1 & PDE3B & RPS6KA3 & TRAF3IP2 & \\
\hline BAG2 & $\overline{C P T 1 B}$ & GINS1 & KIF13B & MED13 & PDLIM5 & RRM1 & TREM1 & \\
\hline BARD1 1 & $C P V L$ & GLI2 & KIF24 & MEF2D & PGAP3 & SARNP & TRIM29 & \\
\hline$B C A N$ & CREB1 & GMIP & KIF2C & MELK & PGM1 & SEC31A & $\overline{T R O V E 2}$ & \\
\hline BMP6 & CTPS & GNB2L1 & KLF16 & MEST & PHKB & SEZ6L2 & TUBB & \\
\hline BPTF & CTSF & GOSR1 & KPNA2 & MFGE8 & $\mathrm{Pl} 3$ & $S F 3 A 2$ & TUSC3 & \\
\hline BTG3 & CUL5 & GPR153 & $\overline{K R A S}$ & MINK & PIAS2 & SFPQ & TXNIP & \\
\hline$\overline{B T N 3} A 3$ & CUL7 & GTF3A & KRT16 & MMP28 & $\overline{P I K 3 C} A$ & SGPP2 & TXNL4A & \\
\hline C10orf116 & $D A A M 1$ & GUCY1A3 & $L A R P$ & $M N X 1$ & PLEKHH1 & SIRT6 & UCN & \\
\hline C13orf1 & DCAF4 & HAS3 & LOC115871 & MOCOS & POLA2 & SLC16A4 & UGT1A10 & \\
\hline C16orf45 & DHFRL1 & HAX1 & LOC120450 & MRPL46 & POLD2 & $S L C 26 A 2$ & UGT2B28 & \\
\hline C1orf63 & DHRS9 & HBA2 & LOC139376 & MRPS6 & POLR2E & SLC29A2 & UGT2B7 & \\
\hline C21orf63 & DICER1 & $H H L$ & LOC81691 & MTAP & PRDM15 & SLC4A7 & UPF1 & \\
\hline$C A 2$ & $D M X L 1$ & $\mathrm{HIBCH}$ & LPHN2 & $M Y C$ & PRKCI & SLC6A8 & VIM & \\
\hline
\end{tabular}

The listed genes are reproducibly deregulated irrespectively on the mutated codon. Note that all the assayed mutations fall within the p53 DNA-binding domain. Bolded genes are in common with the functional assay proposed by Riley et al. ${ }^{3}$ Underlined are genes for which a high-confidence p53 occupancy sites had been mapped. 
Table 5. The tool DAVID groups cluster of genes into biological pathways

\begin{tabular}{llccc}
\hline Category & Term & Count & $\%$ & P-value \\
\hline KEGG_PATHWAY & p53 signaling pathway & 26 & 1.9 & $8.6 \times 10^{-8}$ \\
KEGG_PATHWAY & Metabolism of xenobiotics by cytochrome P450 & 19 & 1.4 & $1.3 \times 10^{-4}$ \\
KEGG_PATHWAY & Progesterone-mediated oocyte maturation & 23 & 1.7 & $3.2 \times 10^{-5}$ \\
KEGG_PATHWAY & Ascorbate and aldarate metabolism & 9 & 0.7 & $3.9 \times 10^{-3}$ \\
KEGG_PATHWAY & Alanine, aspartate and glutamate metabolism & 12 & $3.5 \times 10^{-4}$ & 0.015 \\
KEGG_PATHWAY & Cell cycle & 28 & 0.9 & $5.5 \times 10^{-4}$ \\
KEGG_PATHWAY & Beta-alanine metabolism & 9 & 2 & 0.013 \\
KEGG_PATHWAY & Arginine and proline metabolism & 15 & 0.7 & $2.3 \times 10^{-3}$ \\
\hline
\end{tabular}

The p53 signaling pathway and pathways related to the control of the cell cycle are deregulated, as expected (analysis carried out including all 1846 genes collected among all the published studies).

and its aberrant expression is often linked to a poor prognosis. ${ }^{47}$ Finally, PARD6B was found amplified and overexpressed in a high number of breast cancer cell lines. The encoded protein, PAR6B, has a central role in tight junction assembly, maintenance of cell polarity, all features important for tumor progression and invasion. ${ }^{4}$ Although the precise mechanism at the basis of the upregulation is not established (a loss of transcriptional repression is likely, as stated before), the increase in gene expression could, at least in part, explain some of the novel phenotypes gained by cancer cells, including angiogenesis, drug resistance and altered cell-matrix and cell-cell interactions.

Pathway analysis of deregulated genes using Database for Annotation, Visualization and Integrated Discovery

The possible pathways and biological functions modulated by mutant p53s were evaluated in silico using the tool Database for Annotation, Visualization and Integrated Discovery and in Table 5 the main results (with a KEGG-pathways based analysis) are reported. As expected, the p53 signaling pathway $\left(P=8.6 \times 10^{-8}\right)$, and pathways related to the control of the cell cycle $\left(P=1.3 \times 10^{-3}\right)$, is among the most significant semantic terms. Moreover, an over-representation of genes encoding for enzymes in the metabolism of xenobiotics $\left(P=1.3 \times 10^{-4}\right)$ was found, where, in general, the cytochrome p450 genes are overexpressed. This might be related to the known resistance to chemotherapeutic drugs associated with p53 mutation status of patients' cancer cells. ${ }^{49}$ Intriguingly, an enrichment of deregulated genes in pathways devoted to the catabolism of amino acids was also found (for example, ARG1, arginase; $P R O D H$, proline oxidase; GLS2, glutaminase; GAD1, glutamate decarboxylase 1, all downregulated). The amino-acid catabolism leads to the formation of $\alpha$-ketoglutarate, one of the key substrate for the tricarboxylic acid cycle, which in turn results in enhanced mitochondrial respiration and ATP generation. It is worth to stress here that p53 was shown to have a role not only in the regulation of cell cycle, apoptosis, differentiation, senescence, angiogenesis, ${ }^{50}$ antioxidant response ${ }^{51}$ and glutaminolysis ${ }^{52,53}$ but also in the modulation of both glycolysis $5^{54-56}$ and mitochondrial respiration. ${ }^{57-60}$ Metabolic enzymes including glucose transporters (such as GLUT1 and GLUT4), glycolytic enzymes (such as PGM5 and $H K 2$ ) and tricarboxylic acid cycle enzymes are downstream targets of p53 (ref. 61), and WTp53 was shown to slow the glycolysis. The inhibition of glycolysis can also be achieved by p53-dependent transcriptional activation of synthesis of cytochrome C oxidative 2 , resulting in enhanced mitochondrial respiration. ${ }^{62}$ Thus, mutations within p53 could lead to an increase of the glycolysis, characteristic of cancer cells. ${ }^{63}$ One of the most important genes linking the energy metabolism with p53 was proposed to be GLS2, encoding for glutaminase. It was shown that GLS2 is transactivated by WTp53, and it regulates the cellular energy metabolism by increasing the production of $\alpha$-ketoglutarate, the mithochondrial respiration and the ATP generation. ${ }^{52}$ It is noteworthy that GLS2 expression was shown to be decreased in hepatocellular carcinomas, whereas its overexpression reduced tumor cell colony formation in an in vitro assay. GLS2 downregulation in cancer could be obtained by LOF mutations within p53, and this is consistent with the fact that GLS2 was found among the DMB genes. In addition, PRODH (proline dehydrogenase) was found downregulated in various transcriptome studies (Supplementary Table S1). Interestingly, $P R O D H$ functions as a tumor suppressor, and it suppresses hypoxia-inducible factor signaling by increasing $\alpha$-ketoglutarate. ${ }^{64}$ Thus, overall, p53 mutations could lead to increasing levels of glycolysis and, in parallel, to reduced mitochondrial respiration. This suggests a role of mutant $\mathrm{p} 53 \mathrm{~s}$ in the Warburg effect. The modulation of $\alpha$-ketoglutarate production, through the alteration of amino-acid catabolism, could be one possible mechanism to be considered in a putative p53dependent metabolic shift.

\section{FINAL REMARKS}

As stated before, collected data seem to be in favor of a general lack of activity at the basis of UMB (LOF) and DMB (loss of transcriptional repression) genes. Given that all the p53 mutants taken into consideration here were classified as LOF in in vitro reporter assays, their expected impact corresponds to the lack of the hand in the 'piano model' analogy ${ }^{15}$ and, therefore, the consequence of this gene expression reprogramming could result as a 'sound of silence'. However, several other aspects deserve discussion and open to the possibility of other mechanisms at play. In fact, the majority of p53 mutations encountered within tumors are of missense type. Moreover, tumors commonly retain and overexpress the full-length mutant p53 (ref. 65) and mutations whose effect is a true ablation of the gene sequence, such as large deletions, nonsense substitutions or in/del frameshifts, account for only about $16 \%$ of the cases (www.iarc.fr/p53). This is in striking contrast to the majority of tumor suppressors (for example, RB1, APC, NF1, NF2 and VHL), where the primary mutations are deletion or nonsense, leading to little or no expression of the respective proteins. Dominance or dominantnegative potential of mutant p53s when heterozygous with the WT allele has been considered as an underlying reason for the high preponderance of p53 missense mutations in cancer. However, one should wonder whether the classification of the hotspot mutations as 'inactivating' based on in vitro assays (commonly performed on yeast systems) is completely correct. The fact that missense mutations are preferred to the abrogation of the locus suggests that these two possibilities are not equivalent. p53 knockin mutant mouse models produced an altered tumor spectrum as compared with the knockout models, with more metastatic tumors. ${ }^{6,67}$ Similarly, in an analysis of LiFraumeni patients, germline missense mutations in TP53 have been shown to be associated with an earlier age of onset (9 years) when compared with germline deletions, suggesting a gain-offunction effect of missense p53 mutants in human tumors. ${ }^{68}$ In addition, tumors with mutant p53 proteins may be more 
aggressive (for example, conferring a poor prognosis) as compared with tumors where p53 is lost. ${ }^{18,69-71}$ Thus, it could be hypothesized that these mutations alter profoundly, but not completely abrogate, some of the p53 functions. Various authors suggested a gain-of-function at molecular level for mutant p53s. ${ }^{6,72-76}$ Mutant p53s can directly bind promoters of various targets such as it is for miR-130b, ${ }^{77}$ miR-128-2, ${ }^{78} \mathrm{Axl}^{79}$ or NF-kB2. ${ }^{80}$ It was also shown that they form aberrant protein complexes with interacting partners, such as NF-Y, Sp1, Ets-1 or VDR, perturbing their activity. ${ }^{81}$ More interactions of this type were reported in a recent review. ${ }^{82}$ Among them, it is noteworthy to underline that although WTp53 does not form heterotetramers with $\mathrm{p} 63{ }^{83}$ mutant $\mathrm{p} 53$ s have been shown to bind and sequester TAp63 away from its target genes, hampering its anti-metastatic capacity. ${ }^{84-87}$ Furthermore, other studies showed that mutant p53s bind to p63 and use it as chaperone for the transactivation of novel targets. ${ }^{7}$ In this regard, it should be considered that the enrichment of the degenerated motif detected with COMPASSS within the promoters of $U M B$ genes could be also a marker of the presence of p63-responsive elements, given the sequence similarities with the p53REs. Thus, we cannot rule out that some of the UMB genes are directly/indirectly transactivated by mutant $\mathrm{p} 53 \mathrm{~s}$.

In summary, the elaboration of data already present in the literature allowed to gain novel insights in the biology of p53 and to define novel targets. Further studies are warranted in order to better define the extent of differences in the transactivating activities of different mutant $\mathrm{p} 53 \mathrm{~s}$ and to validate the novel targets suggested here.

\section{CONFLICT OF INTEREST}

The authors declare no conflict of interest.

\section{REFERENCES}

1 Coutts AS, La TN. The p53 response during DNA damage: impact of transcriptional cofactors. Biochem Soc Symp 2006; 73: 181-189.

2 Sermeus A, Michiels C. Reciprocal influence of the p53 and the hypoxic pathways Cell Death Dis 2011; 2: e164.

3 Riley T, Sontag E, Chen P, Levine A. Transcriptional control of human p53-regulated genes. Nat Rev Mol Cell Biol 2008; 9: 402-412.

4 Lane D, Levine A. p53 Research: the past thirty years and the next thirty years. Cold Spring Harb Perspect Biol 2010; 2: a000893.

5 Vousden $\mathrm{KH}$, Prives $\mathrm{C}$. Blinded by the light: the growing complexity of p53. Cell 2009; 137: 413-431.

6 Wiesmuller L. Genetic stabilization by $\mathrm{p} 53$ involves growth regulatory and repair pathways. J Biomed Biotechnol 2001; 1: 7-10.

7 Neilsen PM, Noll JE, Suetani RJ, Schulz RB, Al-Ejeh F, Evdokiou A et al. Mutant p53 uses p63 as a molecular chaperone to alter gene expression and induce a proinvasive secretome. Oncotarget 2011; 2: 1203-1217.

8 Wang B, Xiao Z, Ko HL, Ren EC. The p53 response element and transcriptional repression. Cell Cycle 2010; 9: 870-879.

9 Li FP, Fraumeni Jr. JF, Mulvihill JJ, Blattner WA, Dreyfus MG, Tucker MA et al. A cancer family syndrome in twenty-four kindreds. Cancer Res 1988; 48: 5358-5362.

10 Leroy B, Fournier JL, Ishioka C, Monti P, Inga A, Fronza G et al. The TP53 website: an integrative resource centre for the TP53 mutation database and TP53 mutant analysis. Nucleic Acids Res 2013; 41: D962-D969.

11 Olivier M, Hollstein M, Hainaut P. TP53 mutations in human cancers: origins, consequences, and clinical use. Cold Spring Harb Perspect Biol 2010; 2: a001008.

12 Soussi T, Kato S, Levy PP, Ishioka C. Reassessment of the TP53 mutation database in human disease by data mining with a library of TP53 missense mutations. Hum Mutat 2005; 25: 6-17.

13 Achatz MI, Hainaut P, shton-Prolla P. Highly prevalent TP53 mutation predisposing to many cancers in the Brazilian population: a case for newborn screening? Lancet Oncol 2009; 10: 920-925.

14 Ishioka C, Frebourg T, Yan YX, Vidal M, Friend SH, Schmidt S et al. Screening patients for heterozygous p53 mutations using a functional assay in yeast. Nat Genet 1993; 5: 124-129.

15 Resnick MA, Inga A. Functional mutants of the sequence-specific transcription factor p53 and implications for master genes of diversity. Proc Natl Acad Sci USA 2003; 100: 9934-9939.
16 Menendez D, Inga A, Resnick MA. The biological impact of the human master regulator $\mathrm{p} 53$ can be altered by mutations that change the spectrum and expression of its target genes. Mol Cell Biol 2006; 26: 2297-2308.

17 Rowan S, Ludwig RL, Haupt Y, Bates S, Lu X, Oren M et al. Specific loss of apoptotic but not cell-cycle arrest function in a human tumor derived p53 mutant. EMBO J 1996; 15: 827-838.

18 Acin S, Li Z, Mejia O, Roop DR, El-Naggar AK, Caulin C. Gain-of-function mutant p53 but not p53 deletion promotes head and neck cancer progression in response to oncogenic K-ras. J Pathol 2011; 225: 479-489.

19 Iwakuma T, Lozano G. Crippling p53 activities via knock-in mutations in mouse models. Oncogene 2007; 26: 2177-2184.

20 Smardova J, Smarda J, Koptikova J. Functional analysis of p53 tumor suppressor in yeast. Differentiation 2005; 73: 261-277.

21 Brosh R, Rotter V. When mutants gain new powers: news from the mutant p53 field. Nat Rev Cancer 2009; 9: 701-713.

22 Donzelli S, Biagioni F, Fausti F, Strano S, Fontemaggi G, Blandino G. Oncogenomic approaches in exploring gain of function of mutant p53. Curr Genomics 2008 9: $200-207$

23 Lozano G. The oncogenic roles of p53 mutants in mouse models. Curr Opin Genet Dev 2007; 17: 66-70.

24 Peart MJ, Prives C. Mutant p53 gain of function: the NF-Y connection. Cancer Cell 2006; 10: 173-174.

25 Petitjean A, Achatz MI, Borresen-Dale AL, Hainaut P, Olivier M. TP53 mutations in human cancers: functional selection and impact on cancer prognosis and outcomes. Oncogene 2007; 26: 2157-2165.

26 Song $\mathrm{H}, \mathrm{Xu}$ Y. Gain of function of p53 cancer mutants in disrupting critical DNA damage response pathways. Cell Cycle 2007; 6: 1570-1573.

27 Strano S, Dell'Orso S, Di AS, Fontemaggi G, Sacchi A, Blandino G. Mutant p53: an oncogenic transcription factor. Oncogene 2007; 26: 2212-2219.

28 Blandino G, Deppert W, Hainaut P, Levine A, Lozano G, Olivier M et al. Mutant p53 protein, master regulator of human malignancies: a report on the Fifth Mutant p53 Workshop. Cell Death Differ 2012; 19: 180-183.

29 Hamroun D, Kato S, Ishioka C, Claustres M, Beroud C, Soussi T. The UMD TP53 database and website: update and revisions. Hum Mutat 2006 27: $14-20$.

30 Maccari G, Gemignani F, Landi S. COMPASSS (COMplex PAttern of Sequence Search Software), a simple and effective tool for mining complex motifs in whole genomes. Bioinformatics 2010; 26: 1777-1778.

31 Jordan JJ, Menendez D, Inga A, Noureddine M, Bell DA, Resnick MA. Noncanonical DNA motifs as transactivation targets by wild type and mutant p53. PLoS Genet 2008; 4: e1000104.

32 Menendez D, Inga A, Resnick MA. The expanding universe of p53 targets. Nat Rev Cancer 2009; 9: 724-737.

33 Huang dW, Sherman BT, Lempicki RA. Systematic and integrative analysis of large gene lists using DAVID bioinformatics resources. Nat Protoc 2009; 4: 44-57.

34 Nikulenkov F, Spinnler C, Li H, Tonelli C, Shi Y, Turunen M et al. Insights into p53 transcriptional function via genome-wide chromatin occupancy and gene expression analysis. Cell Death Differ 2012; 19: 1992-2002.

35 Johnson RA, Ince TA, Scotto KW. Transcriptional repression by $\mathrm{p} 53$ through direct binding to a novel DNA element. J Biol Chem 2001; 276: 27716-27720.

36 Budhram-Mahadeo V, Morris PJ, Smith MD, Midgley CA, Boxer LM, Latchman DS. p53 suppresses the activation of the $\mathrm{Bcl}-2$ promoter by the Brn-3a POU family transcription factor. J Biol Chem 1999; 274: 15237-15244.

37 Hoffman WH, Biade S, Zilfou JT, Chen J, Murphy M. Transcriptional repression of the anti-apoptotic survivin gene by wild type p53. J Biol Chem 2002; 277: 3247-3257.

38 Rinn JL, Huarte M. To repress or not to repress: this is the guardian's question. Trends Cell Biol 2011; 21: 344-353.

39 Bohlig L, Rother K. One function--multiple mechanisms: the manifold activities of p53 as a transcriptional repressor. J Biomed Biotechnol 2011; 2011: 464916.

40 Hwang $\mathrm{Cl}$, Matoso A, Corney DC, Flesken-Nikitin A, Korner S, Wang W et al. Wildtype $\mathrm{p} 53$ controls cell motility and invasion by dual regulation of MET expression. Proc Natl Acad Sci USA 2011; 108: 14240-14245.

41 Kobayashi K, Era T, Takebe A, Jakt LM, Nishikawa S. ARID3B induces malignant transformation of mouse embryonic fibroblasts and is strongly associated with malignant neuroblastoma. Cancer Res 2006; 66: 8331-8336.

42 Maltepe E, Keith B, Arsham AM, Brorson JR, Simon MC. The role of ARNT2 in tumor angiogenesis and the neural response to hypoxia. Biochem Biophys Res Commun 2000; 273: 231-238.

43 Kanojia D, Zhou W, Zhang J, Jie C, Lo PK, Wang Q et al. Proteomic profiling of cancer stem cells derived from primary tumors of HER2/Neu transgenic mice. Proteomics 2012; 12: 3407-3415.

44 Pham CG, Bubici C, Zazzeroni F, Papa S, Jones J, Alvarez K et al. Ferritin heavy chain upregulation by NF-kappaB inhibits TNFalpha-induced apoptosis by suppressing reactive oxygen species. Cell 2004; 119: 529-542. 
45 Epsztejn S, Glickstein H, Picard V, Slotki IN, Breuer W, Beaumont C et al. H-ferritin subunit overexpression in erythroid cells reduces the oxidative stress response and induces multidrug resistance properties. Blood 1999; 94: 3593-3603.

46 Liu X, Madhankumar AB, Slagle-Webb B, Sheehan JM, Surguladze N, Connor JR. Heavy chain ferritin siRNA delivered by cationic liposomes increases sensitivity of cancer cells to chemotherapeutic agents. Cancer Res 2011; 71: 2240-2249.

47 Christiansen A, Dyrskjot L. The functional role of the novel biomarker karyopherin alpha 2 (KPNA2) in cancer. Cancer Lett 2013; 331: 18-23.

48 Aranda V, Nolan ME, Muthuswamy SK. Par complex in cancer: a regulator of normal cell polarity joins the dark side. Oncogene 2008; 27: 6878-6887.

49 Lu C, El-Deiry WS. Targeting p53 for enhanced radio- and chemo-sensitivity. Apoptosis 2009; 14: 597-606.

50 Reinhardt HC, Schumacher B. The p53 network: cellular and systemic DNA damage responses in aging and cancer. Trends Genet 2012; 28: 128-136.

51 Budanov AV, Lee JH, Karin M. Stressin' Sestrins take an aging fight. EMBO Mol Med 2010; 2: 388-400.

52 Hu W, Zhang C, Wu R, Sun Y, Levine A, Feng Z. Glutaminase 2, a novel p53 target gene regulating energy metabolism and antioxidant function. Proc Natl Acad Sci USA 2010; 107: 7455-7460.

53 Suzuki S, Tanaka T, Poyurovsky MV, Nagano H, Mayama T, Ohkubo S et al. Phosphate-activated glutaminase (GLS2), a p53-inducible regulator of glutamine metabolism and reactive oxygen species. Proc Natl Acad Sci USA 2010; 107: 7461-7466.

54 Kawauchi K, Araki K, Tobiume K, Tanaka N. p53 regulates glucose metabolism through an IKK-NF-kappaB pathway and inhibits cell transformation. Nat Cell Biol 2008; 10: 611-618.

55 Kawauchi K, Araki K, Tobiume K, Tanaka N. Activated p53 induces NF-kappaB DNA binding but suppresses its transcriptional activation. Biochem Biophys Res Commun 2008; 372: 137-141.

56 Schwartzenberg-Bar-Yoseph F, Armoni M, Karnieli E. The tumor suppressor p53 down-regulates glucose transporters GLUT1 and GLUT4 gene expression. Cancer Res 2004; 64: 2627-2633.

57 Kulawiec M, Ayyasamy V, Singh KK. p53 regulates mtDNA copy number and mitocheckpoint pathway. J Carcinog 2009; 8: 8.

58 Lebedeva MA, Eaton JS, Shadel GS. Loss of p53 causes mitochondrial DNA depletion and altered mitochondrial reactive oxygen species homeostasis. Biochim Biophys Acta 2009; 1787: 328-334.

59 Buzzai M, Jones RG, Amaravadi RK, Lum JJ, DeBerardinis RJ, Zhao F et al. Systemic treatment with the antidiabetic drug metformin selectively impairs p53-deficient tumor cell growth. Cancer Res 2007; 67: 6745-6752.

60 Goldstein I, Ezra O, Rivlin N, Molchadsky A, Madar S, Goldfinger N et al. p53, a novel regulator of lipid metabolism pathways. J Hepatol 2012; 56: 656-662.

$61 \mathrm{Wu}$ W, Zhao S. Metabolic changes in cancer: beyond the Warburg effect. Acta Biochim Biophys Sin (Shanghai) 2013; 45: 18-26.

62 Matoba S, Kang JG, Patino WD, Wragg A, Boehm M, Gavrilova O et al. p53 regulates mitochondrial respiration. Science 2006; 312: 1650-1653.

63 Vousden KH, Ryan KM. p53 and metabolism. Nat Rev Cancer 2009; 9: 691-700

64 Liu W, Phang JM. Proline dehydrogenase (oxidase) in cancer. Biofactors 2012; 38: 398-406.

65 Scian MJ, Stagliano KE, Ellis MA, Hassan S, Bowman M, Miles MF et al. Modulation of gene expression by tumor-derived p53 mutants. Cancer Res 2004; 64: 7447-7454.

66 Jackson EL, Olive KP, Tuveson DA, Bronson R, Crowley D, Brown M et al. The differential effects of mutant $\mathrm{p} 53$ alleles on advanced murine lung cancer. Cancer Res 2005; 65: 10280-10288.

67 Liu DP, Song H, Xu Y. A common gain of function of p53 cancer mutants in inducing genetic instability. Oncogene 2010; 29: 949-956.

68 Bougeard G, Sesboue R, Baert-Desurmont S, Vasseur S, Martin C, Tinat J et al. Molecular basis of the Li-Fraumeni syndrome: an update from the French LFS families. J Med Genet 2008; 45: 535-538.

69 Solomon H, Madar S, Rotter V. Mutant p53 gain of function is interwoven into the hallmarks of cancer. J Pathol 2011; 225: 475-478.

70 Ushio Y, Tada K, Shiraishi S, Kamiryo T, Shinojima N, Kochi M et al. Correlation of molecular genetic analysis of p53, MDM2, p16, PTEN, and EGFR and survival of patients with anaplastic astrocytoma and glioblastoma. Front Biosci 2003; 8: e281-e288.

71 van Slooten HJ, van De Vijver MJ, Borresen AL, Eyfjord JE, Valgardsdottir R, Scherneck $S$ et al. Mutations in exons 5-8 of the p53 gene, independent of their type and location, are associated with increased apoptosis and mitosis in invasive breast carcinoma. J Pathol 1999; 189: 504-513.

72 Greenblatt MS, Bennett WP, Hollstein M, Harris CC. Mutations in the p53 tumor suppressor gene: clues to cancer etiology and molecular pathogenesis. Cancer Res 1994; 54: 4855-4878.

73 Kremenetskaya OS, Logacheva NP, Baryshnikov AY, Chumakov PM, Kopnin BP. Distinct effects of various p53 mutants on differentiation and viability of human K562 leukemia cells. Oncol Res 1997; 9: 155-166.
74 Cadwell C, Zambetti GP. The effects of wild-type p53 tumor suppressor activity and mutant p53 gain-of-function on cell growth. Gene 2001; 277: 15-30.

75 Lin J, Teresky AK, Levine AJ. Two critical hydrophobic amino acids in the $\mathrm{N}$-terminal domain of the $\mathrm{p} 53$ protein are required for the gain of function phenotypes of human p53 mutants. Oncogene 1995; 10: 2387-2390.

76 Sigal A, Rotter V. Oncogenic mutations of the 553 tumor suppressor: the demons of the guardian of the genome. Cancer Res 2000; 60: 6788-6793.

77 Dong P, Karaayvaz M, Jia N, Kaneuchi M, Hamada J, Watari H et al. Mutant p53 gain-of-function induces epithelial-mesenchymal transition through modulation of the miR-130b-ZEB1 axis. Oncogene 2012; 32: 3286-3295.

78 Donzelli S, Fontemaggi G, Fazi F, Di AS, Padula F, Biagioni F et al. MicroRNA-128-2 targets the transcriptional repressor E2F5 enhancing mutant p53 gain of function. Cell Death Differ 2012; 19: 1038-1048.

79 Vaughan CA, Frum R, Pearsall I, Singh S, Windle B, Yeudall A et al. Allele specific gain-of-function activity of p53 mutants in lung cancer cells. Biochem Biophys Res Commun 2012; 428: 6-10.

80 Vaughan CA, Singh S, Windle B, Sankala HM, Graves PR, Andrew YW et al. p53 mutants induce transcription of NF-kappaB2 in H1299 cells through CBP and STAT binding on the NF-kappaB2 promoter and gain of function activity. Arch Biochem Biophys 2012; 518: 79-88.

81 Gurtner A, Starace G, Norelli G, Piaggio G, Sacchi A, Bossi G. Mutant p53-induced up-regulation of mitogen-activated protein kinase kinase 3 contributes to gain of function. J Biol Chem 2010; 285: 14160-14169.

82 Freed-Pastor WA, Prives C. Mutant p53: one name, many proteins. Genes Dev 2012; 26: 1268-1286.

83 Davison TS, Vagner C, Kaghad M, Ayed A, Caput D, Arrowsmith CH. p73 and p63 are homotetramers capable of weak heterotypic interactions with each other but not with p53. J Biol Chem 1999; 274: 18709-18714.

84 Irwin MS, Kondo K, Marin MC, Cheng LS, Hahn WC, Kaelin Jr WG. Chemosensitivity linked to p73 function. Cancer Cell 2003; 3: 403-410.

85 Melino G. p63 is a suppressor of tumorigenesis and metastasis interacting with mutant p53. Cell Death Differ 2011; 18: 1487-1499.

86 Strano S, Rossi M, Fontemaggi G, Munarriz E, Soddu S, Sacchi A et al. From p63 to p53 across p73. FEBS Lett 2001; 490: 163-170.

87 Strano S, Fontemaggi G, Costanzo A, Rizzo MG, Monti O, Baccarini A et al. Physical interaction with human tumor-derived p53 mutants inhibits p63 activities. J Biol Chem 2002; 277: 18817-18826.

$88 \mathrm{Li} \mathrm{CQ}$, Robles Al, Hanigan CL, Hofseth L, Trudel L, Harris CC et al. Apoptotic signaling pathways induced by nitric oxide in human lymphoblastoid cells expressing wild-type or mutant p53. Cancer Res 2004; 64: 3022-3029.

89 O'Farrell TJ, Ghosh P, Dobashi N, Sasaki CY, Longo DL. Comparison of the effect of mutant and wild-type p53 on global gene expression. Cancer Res 2004; 64: 8199-8207.

90 Bossi G, Marampon F, Maor-Aloni R, Zani B, Rotter V, Oren M et al. Conditional RNA interference in vivo to study mutant $\mathrm{p} 53$ oncogenic gain of function on tumor malignancy. Cell Cycle 2008; 7: 1870-1879.

91 Junk DJ, Vrba L, Watts GS, Oshiro MM, Martinez JD, Futscher BW. Different mutant/wild-type p53 combinations cause a spectrum of increased invasive potential in nonmalignant immortalized human mammary epithelial cells. Neoplasia 2008; 10: 450-461.

92 Mizuarai S, Yamanaka K, Kotani H. Mutant p53 induces the GEF-H1 oncogene, a guanine nucleotide exchange factor- $\mathrm{H} 1$ for RhoA, resulting in accelerated cell proliferation in tumor cells. Cancer Res 2006; 66: 6319-6326.

93 Tepper CG, Gregg JP, Shi XB, Vinall RL, Baron CA, Ryan PE et al. Profiling of gene expression changes caused by $p 53$ gain-of-function mutant alleles in prostate cancer cells. Prostate 2005; 65: 375-389.

94 Weisz L, Zalcenstein A, Stambolsky P, Cohen $\mathrm{Y}$, Goldfinger $\mathrm{N}$, Oren $\mathrm{M}$ et al. Transactivation of the EGR1 gene contributes to mutant p53 gain of function. Cancer Res 2004; 64: 8318-8327.

95 Scian MJ, Stagliano KE, Deb D, Ellis MA, Carchman EH, Das A et al. Tumor-derived p53 mutants induce oncogenesis by transactivating growth-promoting genes. Oncogene 2004; 23: 4430-4443.

96 Scian MJ, Stagliano KE, Anderson MA, Hassan S, Bowman M, Miles MF et al. Tumor-derived p53 mutants induce NF-kappaB2 gene expression. Mol Cell Biol 2005; 25: 10097-10110.

(c) (i) $\Theta$ Oncogenesis is an open-access journal published by Nature Publishing By NC ND Group. This work is licensed under a Creative Commons AttributionNonCommercial-NoDerivs 3.0 Unported License. To view a copy of this license, visit http://creativecommons.org/licenses/by-nc-nd/3.0/

Supplementary Information accompanies this paper on the Oncogenesis website (http://www.nature.com/oncsis). 